\title{
Detlef D. Spalt: Die Analysis im Wandel und im Widerstreit. Eine Formierungsgeschichte ihrer Grundbegriffe
}

\author{
Verlag Karl Alber, 2015, xxvii+762 Seiten, ISBN: \\ 978-3-495-48740-2
}

\section{Thomas Sonar}

Online publiziert: 17. März 2017

(C) Der/die Autor(en) 2017. Dieser Artikel ist eine Open-Access-Publikation.

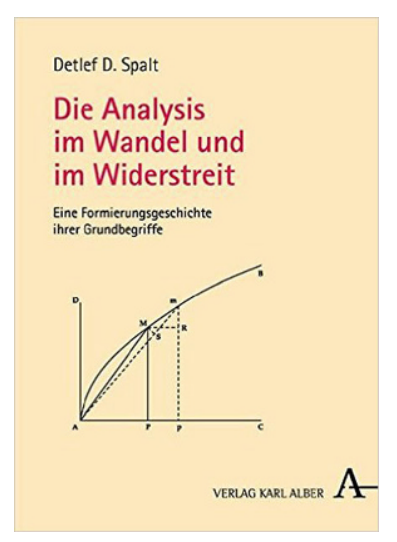

Detlef Spalt hat sich zum Ziel gesetzt, die Geschichte der Analysis aus den Quellen heraus verstehen und präsentieren zu wollen, und allein diese Zielsetzung ist ihm hoch anzurechen. In den letzten Jahren hat sich die Interpretation einer Geschichte der Mathematik in unserem Land stark gewandelt; man diskutiert gerne „,kontextuell“, was das genaue Studium der Quellen häufig leider verdrängt hat. Gleich zu Anfang weist Spalt auf die inhärenten Schwierigkeiten der quellenbezogen Arbeit hin: die Alten haben sich anders ausgedrückt als wir und gewisse Bedeutungen haben sich im Laufe der Jahrhunderte verschoben. Ja, das ist wahr, und jeder, der die Quellen liest, transkribiert, übersetzt und vielleicht dann noch interpretiert tut gut daran, dieses Problem im Hinterkopf zu behalten. Eine etwas steile These findet sich gleich zu Anfang des Buches, nämlich die Behauptung, es hätte sich bisher noch niemand für die Erforschung der Probleme von „Geist und Materie, Möglichkeit und Wirklichkeit, Sinn und Bedeutung mathematischer Begriffe" im Zusammenhang mit der Geschichte der Analysis beschäftigt. Einem solchen Diktum ist unter dem Eindruck der zahlreichen Arbeiten von Eberhard Knobloch, Rüdiger Thiele, Niccolò Guicciardini, Jacqueline Stedall und vieler anderer Historiker der Analysis nur schwer zu folgen. Auch die These, die Formierungsgeschichte der Analysis beginne vor nicht mehr als 400 Jahren, kann man entweder akzeptieren oder nicht, denn auch Newton bezog sich gerne auf Pappos von Alexandrien und entlehnte von ihm gewisse Begriffe und

T. Sonar $(\triangle)$

Technische Universität Braunschweig, Braunschweig, Deutschland

E-Mail: t.sonar@tu-bs.de 
Vorgehensweisen [2]; die Leistungen von Archimedes würde man vielleicht auch gerne zu den Errungenschaften der Analysis rechnen, wie es z.B. Edwards [1] tut.

Ein wichtiger Begriff, zu dessen Aufklärung der Autor beitragen will, ist der Begriff der „Größe“, wie er seit Newton und Leibniz bis ins 19. Jahrhundert hinein gebräuchlich war. Allerdings ist die Situation nicht ganz so ein ,offenkundiger blinder Fleck“ (S. xxi) wie der Autor meint. Dass der Autor ein streitbarer Mathematikhistoriker ist, hat er in der Vergangenheit immer wieder bewiesen, aber die Behauptung auf S. xxi, in meinem Buch zur Geschichte der Analysis [3] sei der Begriff „Größe“ nicht einmal im Register vorhanden, ist schon ein starkes Stück. Auf Seite 698 findet man im Sachverzeichnis von [3] den Indexeintrag „Größen“ und dazu 10 Unterkategorien. Andere Autoren kommen ebenfalls nicht gut weg, aber das soll den Gesamteindruck des ansonsten sehr empfehlenswerten Werkes nicht schmälern.

Das erste Kapitel behandelt die Erfindung der formalen Algebra durch Descartes; ohne Zweifel eine geistige Meisterleistung, die der Mathematik in der Tat eine ganz neue Sprache gab. Der Autor beginnt allerdings mit Galiei und seiner ,Sprache der Mathematik“, die ganz in geometrischen Figuren verhaftet blieb. Und hier zeigt sich ganz klar die Spalt'sche Stoßrichtung: Galileis Satz über die Sprache der Mathematik hat sich durch Descartes gewandelt, von geometrischen Figuren hin zu einer Symbolsprache. Behält man dieses erste Beispiel im Gedächtnis, dann öffnet sich der Sinn der Untersuchungen des Autors leichter, der schon im Titel enthalten ist: „Die Analysis im Wandel ...“. Spalt legt immer großen Wert auf philosophische Interpretationen; so auch im Fall von Descartes (S. 63f.). Immer findet man gerade in diesen Interpretationen interessante Einsichten und Thesen; ob die Leserin oder der Leser sich diesen immer anschließen mag, wird von der jeweiligen weltanschaulichen Inklination abhängen - anregend sind die Spalt'schen Ausflüge immer.

Im zweiten Kapitel geht es um die Erfindung der stetig Veränderlichen durch Leibniz. Es beginnt mit einer Begriffslücke, die Descartes bei der Behandlung von Gleichungen gelassen hat. Er löste Gleichungen, aber der „Punkt“ als Lösung der Gleichung ist noch keine Linie, von der Descartes aber ansonsten spricht. Spalt schreibt (S. 67): „Doch eine Gleichung „zeichnet“ keine Linie.“ Spalt analysiert diese Begriffslücke detailiert und blendet dann auf Leibniz, dessen Erfindung der veränderlichen Größe diese Lücke schließt. Spalt diskutiert auch detailliert den ersten allgemeinen Konvergenzbegriff Leibnizens im Manuskript De quadratura arithmetica. Das Manuskript wurde erst 1993 vollständig durch Eberhard Knobloch transkribiert und liegt heute in einer deutschen Übersetzung vor. Auch auf den Leibniz'schen Differentialkalkül wird eingegangen, auf die characteristica universalis und die Monadenlehre, wodurch ein sehr ,rundes“ Bild der mathematischen Leistungen Leibnizens entsteht. In historiographischen Nachträgen werden die Indivisiblenrechnung, die Stetigkeit des Kontinuums und Newtons Fluxionenrechnung behandelt. Auch hier finden sich kluge Überlegungen des Autors, um die sauber beschriebenen mathematischen Errungenschaften historisch und philosophisch einzuordnen.

Das dritte Kapitel trägt den Titel „Grundlagen der Algebraischen Analysis“. Es startet mit Johann Bernoulli und dessen Auffassung des Leibniz'schen Differentialkalküls. Spalt legt dar, dass Bernoulli sich von den metaphysischen „Scheuklappen“ 
(S. 187) Leibnizens freimachte und ein „Unendlich“ als etwas Konkretes ansah. Bernoulli versuchte auch, Leibnizens geometrische Begründungen des Kalküls in algebraischer Form zu fassen, was Spalt hervorragend darlegt und einer kritischen Analyse unterzieht. L'Hospital setzte dann in seinem berühmten Lehrbuch die Bernoulli'sche Auffassung des Leibniz'schen Kalküls um. In der Generation nach Bernoulli ist es dessen Schüler Euler, der die Ideen aufnimmt und weiter ausbaut und entwickelt. Sehr genau diskutiert Spalt die Genese des Funktionsbegriffs, der bei Johann Bernoulli seinen Anfang nahm. Mit dem Funktionsbegriff bei Euler beschäftigt sich der Autor bereits Jahren und so nimmt es nicht Wunder, dass die sich damit befassenden Abschnitte des Buches sehr umfangreich sind. Dass Euler zwei Notationen für Funktionen verwendete ist interessant; interessanter finde ich die Frage nach der Bedeutung des Begriffs „Größe“ bei Euler, der Spalt mit detektivischem Scharfsinn nachspürt. Die Algebraische Analysis wird von Lagrange weiter entwickelt und in gewisser Weise zu einem Höhepunkt gebracht. Spalt analysiert die dort verwendeten Begriffe sehr genau und berichtet auch über die aufkommende Kritik.

Dann wandeln sich die Dinge und in Kapitel 4 geht es um die Begründung der „Werte-Analysis“: Bolzanos Programm wird analysiert und Cauchys Cours d'Analyse bricht endgültig mit der Analysis der „Größen“ und etabliert eine Analysis der ,Werte“, was eine Umdeutung der bisherigen Begriffe der Infinitesimalmathematik erfordert, die Spalt genau untersucht. Es hat vor einigen Jahren eine Kontroverse um die Interpretation unendlich kleiner Größen bei Cauchy zwischen Spalt und einigen anderen Mathematikhistorikern gegeben, die nicht immer sehr zivilisiert geführt wurde. Hier nun stellt Spalt seine Auffassung noch einmal klar und bemerkt natürlich auch, dass seine Studie hier quer zum „Mainstream“ liegt. Sehr schade finde ich, dass Spalt bei der Diskussion der Kontroverse auf Seite 313 f. verbal sehr um sich schlägt. Er wirft anderen Mathematikhistorikern vor, nicht „verständig lesen“ zu können und bezeichnet die Gegner seiner eigenen Auffassung in Fußnote 224 sogar als ,wissenschaftliche Scharlatane“. Ich maße mir kein Urteil darüber an, wer in der Kontroverse „Recht“ hat, aber das vorliegende Buch hat polemische Ausbrüche dieser Art einfach nicht verdient - sie erscheinen mir fehl am Platz und gehen so weit, die heutige Wissenschaft generell als ,zensierend“ zu bezeichnen (S. 314 unten). Weiter geht es bei der Diskussion um den Cauchy'schen „Summensatz“ und die Bedeutung des Wortes ,,convergent“. Dazu hat Spalt bereits in der Vergangenheit gearbeitet, vergl. [4] und die jüngere Publikation [5]. Man kann sich Spalts Interpretationen anschließen, man muss es jedoch nicht. Zu diesem Resultat kam auch Ernst Specker in einer Buchbesprechung von [4] für die Naturforschende Gesellschaft in Zürich Spalts Interpretationen bleiben jedenfalls interessant.

Im fünften Kapitel beschreibt Spalt das ,,analytische Interregnum von 1817 bis 1872“, das auf Cauchys radikal neue Analysis folgte. Die Unsicherheiten beim Begriff des Funktionswerts werden aufgedeckt und analysiert, Riemanns grandiose Leistungen, die schließlich den Weg zur Mengenlehre öffnen, werden beleuchtet, und schließlich geht Spalt auf Weierstraß und sein „Ringen um die Grundbegriffe der Analysis“ ein. Auch hier bleibt der Autor dem Leitthema „Wandel“ im Titel des Buches treu. Wieder bekommen einige Autoren ,ihr Fett weg“ (so auch ich), aber der Ton bleibt im Gegensatz zum vierten Kapitel gemäßigt. 
Das Kapitel 6, „Konsolidierung (1)“ ist der Erfindung der reellen Zahlen 1872 i.w. durch Cantor und ihrer Vorgeschichte gewidmet. Die komplizierten und teilweise unübersichtlichen Zusammenhänge der verschiedenen Kritiker Cantors (wie etwa Frege) werden entwirrt, Dedekinds Beiträge genau analysiert, und das Problem der „Stetigkeit der Linie“ genau beleuchtet. Ein großes Plus dieses Buches, das sich auch in diesem Kapitel zeigt, sind die „Reflexionen“, die Spalt immer wieder einschiebt, um zusammenzufassen, Standpunkte zu klären, und eigene Interpretationen zu geben. Auch die Laugwitz/Schmieden'schen --Zahlen werden hier behandelt als Konstruktion eines Zahlbegriffs, der auf der Grundlage nicht konvergierender Folgen basiert. Hier befindet sich Spalt ganz in seinem Element, denn er ist sicher unangefochten der führende Mathematikhistoriker, der die Entwicklung dieses Kalküls besser kennt als irgend jemand sonst. Zur Diskussion des Zahlbegriffs (besser: der Zahlbegriffe) gehört auch eine Diskussion der philosophischen Grundlagen und Spalt liefert auch diese via Husserls intensionaler Fassung des Zahlbegriffs. Das Kapitel endet mit Hilberts axiomatischer Methode und einer bemerkenswerten Diskussion ihrer Folgen.

Im siebten Kapitel, „Konsolidierung (2)“, geht es wieder verstärkt um den Funktionsbegriff. Es beginnt mit Heine, vergleicht die Funktionsbegriffe von Weierstraß und Riemann, und folgt der Tradition der deutschsprachigen Literatur, die sich an Riemann orientiert. Die französische Tradition wird ebenfalls diskutiert, dann Kleins Auffassung der Mathematik als Theorie der Naturerscheinungen, an Hand derer Spalt den Stand der Diskussion bis zum Beginn des 20. Jahrhunderts analysiert. „Funktion“ ist bis zu diesem Zeitpunkt noch nicht mit einer sauberen, inhaltlichen Definition versehen. Weierstraß hatte das gefordert, aber die Mathematiker folgten Riemann. Nun kommt mit der Mengenlehre Cantors eine ontologische Neubestimmung, in dem der „Funktion“ die Beschreibung als „Menge“ beigegeben wird. Hausdorff verhalf dieser Auffassung zum Durchbruch, aber die Lehrbuchliteratur brauchte noch lange, ehe der Mengenbegriff sich auch dort durchsetzte.

Das Buch endet mit einem „Ausklang“ zur Bedeutung des aktualen Unendlich als philosophischem Joker in der heutigen Mathematik. Ein umfangreiches Literaturverzeichnis, ein Personenregister und ein Sachregister beschließen das Werk.

Ich kann das Werk von Spalt nur wärmstens empfehlen. Es ist in seiner Art in der deutschen Literatur (und sicher nicht nur dort) einzigartig und gibt detaillierte Einblicke nicht nur in die Entwicklung der Analysis und ihrer Begriffe, sondern enthält auch reichhaltige philosophische Diskussionen und Interpretationen, die man in anderen Werken in dieser Form nicht findet. Ob man sich den Interpretationen in jedem Fall anschließen möchte, sollten die Leserinnen und Leser für sich entscheiden; am besten, wenn sie selbst einmal die Quellen studieren würden, was sicherlich auch im Sinne des Autors wäre. Dass das Werk in einigen Teilen scharfe Polemik enthält, die in einem Buch zur Geschichte der Analysis meiner Meinung nach nichts zu suchen hat, ist sehr bedauerlich und trübt den ansonsten sehr positiven Eindruck ein wenig. 
Open Access Dieser Artikel wird unter der Creative Commons Namensnennung 4.0 International Lizenz (http://creativecommons.org/licenses/by/4.0/deed.de) veröffentlicht, welche die Nutzung, Vervielfältigung, Bearbeitung, Verbreitung und Wiedergabe in jeglichem Medium und Format erlaubt, sofern Sie den/die ursprünglichen Autor(en) und die Quelle ordnungsgemäß nennen, einen Link zur Creative Commons Lizenz beifügen und angeben, ob Änderungen vorgenommen wurden.

\section{Literatur}

1. Edwards Jr., C.H.: The historical development of the calculus. Springer, Berlin (1979)

2. Guicciardini, N.: Isaac Newton on mathematical certainty and method. MIT Press, Cambridge (2011)

3. Sonar, T.: 3000 Jahre Analysis. Springer, Berlin (2011)

4. Spalt, D.: Die Vernunft im Cauchy-Mythos. Harri Deutsch, Frankfurt am Main (1996)

5. Spalt, D.: Cauchys Kontinuum. Eine historiographische Annäherung via Cauchys Summensatz. Arch Hist Exact Sci 56, 285-338 (2002) 